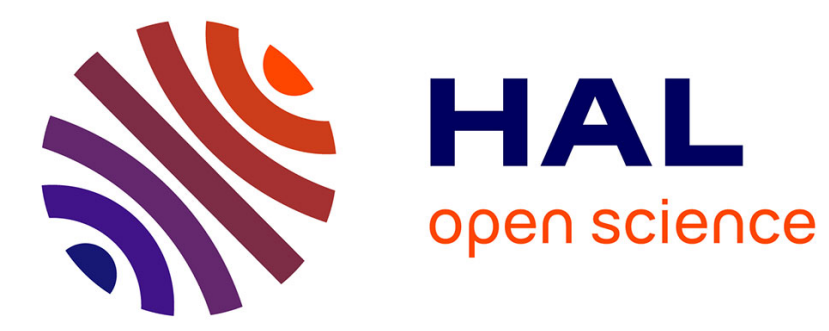

\title{
Digital twins: properties, software frameworks, and application scenarios
}

\author{
Roberto Minerva, Noel Crespi
}

\section{To cite this version:}

Roberto Minerva, Noel Crespi. Digital twins: properties, software frameworks, and application scenarios. IT Professional, 2021, 23 (1), pp.51 - 55. 10.1109/mitp.2020.2982896 . hal-03210180

\section{HAL Id: hal-03210180 \\ https://hal.science/hal-03210180}

Submitted on 27 Apr 2021

HAL is a multi-disciplinary open access archive for the deposit and dissemination of scientific research documents, whether they are published or not. The documents may come from teaching and research institutions in France or abroad, or from public or private research centers.
L'archive ouverte pluridisciplinaire HAL, est destinée au dépôt et à la diffusion de documents scientifiques de niveau recherche, publiés ou non, émanant des établissements d'enseignement et de recherche français ou étrangers, des laboratoires publics ou privés. 


\section{General Interest}

1

2
The digital twin (DT) was conceived to design, prototype, and operate a virtual copy of a product. ${ }^{1}$ It promotes the softwarization of physical objects (POs), into logical components and the development of applications that exploit programmability of the software representation. Originally used by $\mathrm{NASA}^{2}{ }^{2}$ it has gained interest in manufacturing. ${ }^{3}$ Because of its ability to describe POs by means of logical models and actual data, it is increasingly used in the Internet of Things and cyber-physical

Digital Object Identifier 10.1109/MITP.2020.2982896 systems. ${ }^{4}$ To fully master its potential, there is a need to understand what a DT is from a software perspective, how to support and implement it, how to manage the physical and logical counterparts, and what constraints are implied by the relationship between them. This article addresses the current DT definition, and identifies a set of characterizing properties. Next, a software framework is sketched out to illustrate two application scenarios that highlight technical possibilities and challenges. An analysis of some business benefits is provided. Conclusions summarize challenges and risks of this approach. 


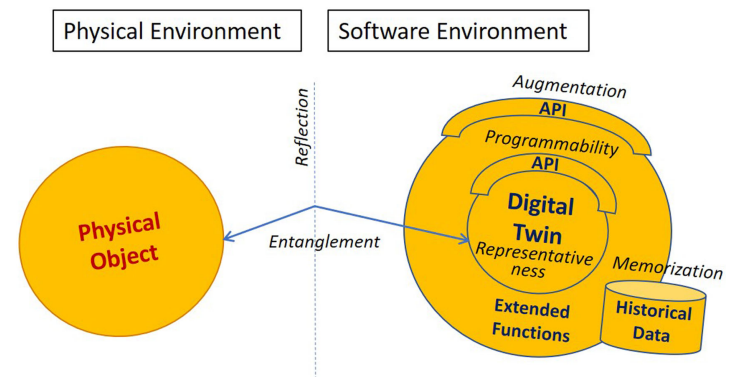

Figure 1. DT representation.

PROPERTIES

DTs embody software capabilities to describe or simulate relevant features and behavior of POs from design to end-of-life phases. ${ }^{5}$ A DT is a software actionable representation of a PO (or aggregated objects) in a specific environment. Some PO's features could be unrelated to the goals of the software representation. Products or complex objects are the main targets, because mapping one to one atomic objects may be unpractical or useless. DTs in a cyber-physical systems (CPS) domain are characterized ${ }^{6}$ by events they are capable of managing, methods and functionalities they offer, storage capabilities to maintain a historical log of status changes, ability to serve as functional interfaces to applications through application programming interfaces (APIs) and ability to send/receive information. The definition of basic DT properties (see Figure 1) is instrumental to determine requirements and constraints posed to software platforms. The key properties of a DT are given as follows.

- Representativeness and contextualization. A DT represents the $\mathrm{PO}$ in a specific context, i.e., a DT is a software implementation of a model representing the PO in a specific environment. Feature simplifications or generalizations are possible when they do not affect the behavior in the specific context.

- Reflection. A DT mirrors the behavior and the status of the PO. Each change in status, each event faced by the PO is reflected by the DT. Changes that occur to the DT should be reproduced in the $\mathrm{PO}$.

- Entanglement. A DT and its PO must be constantly "connected" to instantaneously (i.e., in a time period consistent with requirements of applications) register any change in status. 79 Entanglement requires an effective, reliable 80 communication between the PO and the DT 81 suitable to the rate of changes.

- Persistency. A DT is always available. Its avail- 83 ability exceeds the actual existence of the 84 PO. A DT could be available before the 85 "creation," during malfunctioning and 86 crashes, and after the end of life of the PO. $\quad 87$

- Memorization. A DT stores all the status 88 changes and events occurred to the PO. A DT 89 represents the status of the $\mathrm{PO}$ over time 90 and space. tions and offer them by means of APIs. Aug- 93 mentation can add new functionalities that 94 the PO does not support or provide access 95 to data in particular formats.

96

- Composability. Physical systems are aggrega- 97 tions of subsystems and components, a DT 98 must support the correlation of different ele- 99 mentary DTs into complex organizations and 100 provide views on the aggregated DT and indi- 101 vidual components. 102

- Replication. A DT can be replicated to serve 103 the needs of different applications. Replicas 104 of the same PO must behave consistently, 105 i.e., they cannot have a different status and 106 they cannot exhibit different behaviors. $\quad 107$

- Accountability/Manageability. Each DT must 108 be manageable; it is possible to determine its 109 status and activities and to optimize its exe- 110 cution in the framework in which it is operat- 111 ing. It must provide information about the 112 usage of the PO by the applications associ- 113 ated with it. Self-management of DTs is a 114 desirable property for large systems. 115

- Servitization. ADT enables the transformation 116 of a product/artifact into a set of functionali- 117 ties offered to users. This capability trans- 118 forms products into software-controlled and 119 connected entities accessible "on-demand" 120 by users.

- Predictability. ADT has the ability to simulate its 122 behavior over time. Specific instances of DTs 123 can simulate the behavior of PO in a context at 124 a specific time (in the past or in the future). $\quad 125$

Some DT properties need contextualization: 126 replicas are used for tracking POs or for simulate 127
- Augmentation. A DT can extend the PO func- 92 


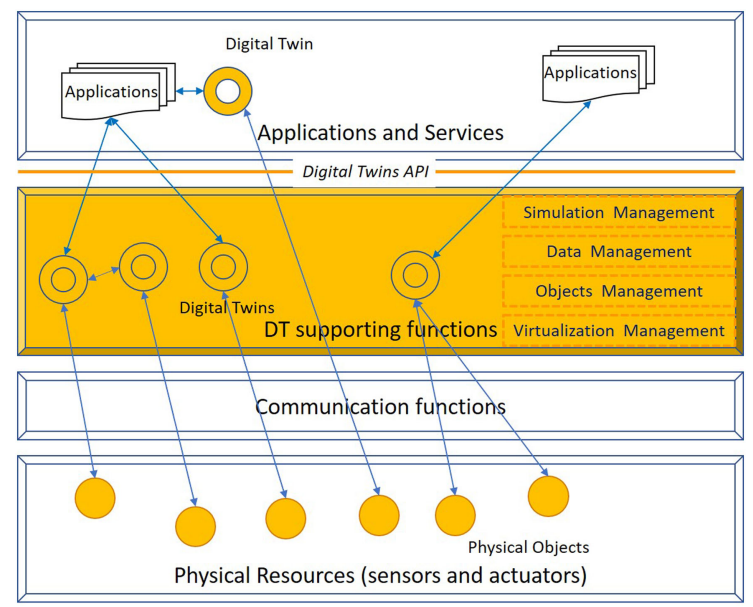

Figure 2. General framework for digital twins.

their behavior. All the replicas of a DT tracking the actual status of a PO should have the same status at the same time $t$ (with time limitations explained for entanglement), DTs used for simulations can diverge depending on the simulation conditions.

\section{TOWARDS A GENERAL SOFTWARE FRAMEWORK}

A software framework for DTs implements numerous functionalities, e.g., orchestration of objects, cognition for intelligent optimization, self-management to minimize human intervention. Different software architectures may be applicable. ${ }^{7,8}$ Some adopt layering principles for organizing functionalities. ${ }^{9}$ These architectures must be evaluated to determine how properly they support the DT requirements. Figure 2 represents a generic framework organized in four functional segments. This structure is chosen to illustrate the separation of concerns. IoT devices, communications means (protocols and networks), DT tools and functions will quickly evolve. Layering may prove useful: it is simple enough to be practically used and can allow independent evolution of the different technological layers. Finally, layering is aligned with efforts in standardizing IoT architectures. AIOTI ${ }^{10}$ describes an IoT layered architecture that could support the DT approach. However, modularization and linkage of functionalities can prove as practical as layered architectures. Physical resources are devices producing information or acting on the environment (sensors and actuators). The information (commands, events, requests, and status notifications) exchanged between devices and components of the framework is transported by means of communications functions. The DT supporting functions segment represents the execution environment of DTs and the set of intelligent functionalities that DT instances use for orchestrating their interactions and lifecycles (e.g., instantiation, replication, identification, access control, and self-management), managing collected data, and simulation capabilities. This segment mediates the interaction between applications and physical devices. New functions, mechanisms and tools may be progressively designed and added to better meet DTs requirements. The application and services segment supports the applications that use the DTs interfaces and functions. For customization and execution purposes, DT replicas may be locally executed in this environment.

The actual exploitation of the DT as a generalpurpose approach occurs if some conditions are met: the availability of a software framework capable of fulfilling the properties of DTs; the ability to create new applications with better functionalities for the user; and the ability to introduce new business models or to improve existing ones. These issues are analyzed in the following sections.

\section{APPLICATION SCENARIOS}

DT can be used to implement several scenarios. In this article, two application scenarios, based on properties of DTs and software framework, illustrate possibilities and issues.

\section{Scenario 1: Virtual Object}

This example describes how to create the DT of an object. A sensor is chosen, but different (aggregations of) objects may be considered. The needed design tasks are given as follows.

Physical object model: An abstract computable description of the data and behavior of the PO that the DT implements. The sensor status and the measurements it takes in the environment are relevant aspects to consider and to model.

API definition: an interface for requesting the functionalities offered by the DT. Applications use it to get data or to give commands. Data formats and interaction mechanisms should be defined. 
Entanglement model: a model governing how PO and DT exchange data and status updates. The framework must offer mechanisms and protocols to support communication. Often the data flow is unidirectional from PO to DT, while it is bidirectional between the DT and applications (data, events, and commands). Commands from the applications to DT are used to govern the DT behavior.

A single PO can be replicated by several DTs. This occurrence accommodates specific requirements of applications: providing special functions, or permit access to the data with different rates, or segmenting and securing the computational space. Replicas can be instantiated in the framework and others in the application space. This option offers flexibility in deployment. Replication of DTs poses interesting issues. When the status of the PO changes, all the replicas need to change status. The propagation of information can be achieved with different mechanisms; PubSub is used in IoT implementations ${ }^{11}$ and it suits the case of unidirectional communication (where the PO is a publisher and the replicas are subscribers). If $\mathrm{PO}$ status can be modified or it can be instructed (an actuator), the problem of synchronization and consistency of information between the PO and different replicas emerges. This issue can be solved by introducing transaction management functions into the framework.

\section{Scenario 2: Digital Twins in Smart City}

Cities are complex systems ${ }^{12}$ difficult to control and program. A prerequisite for a smart city is having "measurable" attributes. Sensors and actuators are deployed to capture data that describe its processes. This make a city quantifiable and assessable. Once reliable data are available, artificial intelligence and machine learning, $\mathrm{AI} / \mathrm{ML}$, techniques determine patterns and behavioral models of urban systems. ${ }^{13}$ The city becomes "understandable," i.e., it is possible to predict events and their associated causes by means of data analysis. DTs are a step toward the programmability of a city: the digital representation of resources makes it possible to design applications that, by interacting with DTs, can affect the behavior of "things" and optimize city resources and their behavior. Several experiences introduce the DT concept in smart

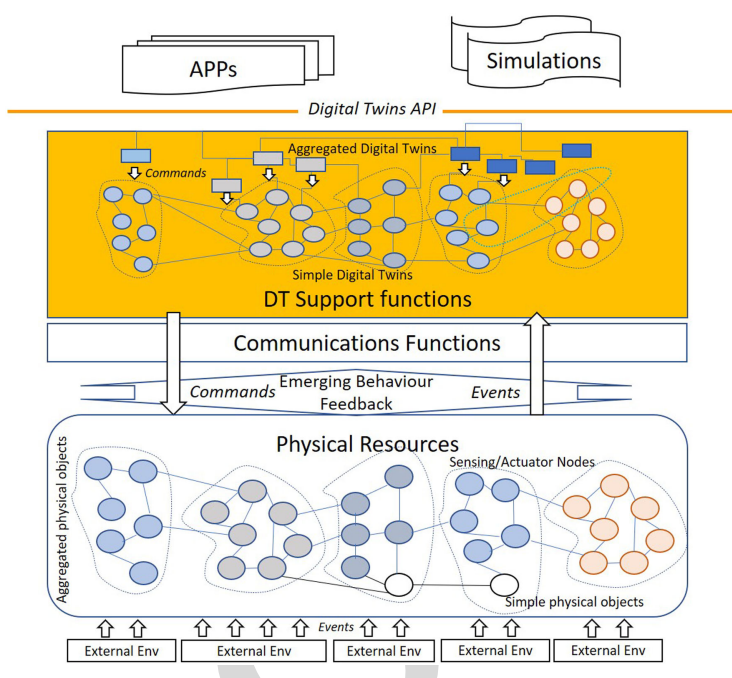

Figure 3. Programmability of complex systems with digital twins

cities. $^{14,15}$ The DT application introduces new 259 capabilities and has an impact on how to govern 260 the complexity of urban systems. For exam- 261 ple, the adoption of the DT approach is improv- 262 ing the usage of building information modeling, 263 e.g., such as given by Delbrügger et al. ${ }^{16} \quad 264$

DTs are programmable objects that can 265 orchestrate, on request of applications, POs like 266 actuators toward the desired behavior. DTs can 267 detect malfunctioning in POs and minimize their 268 impact on other resources. DTs, exploiting 269 advancement in storage technology, can store 270 large datasets and hence supporting the continu- 271 ous "learning" of city behavior. They offer the 272 possibility to evaluate new policies by simulat- 273 ing future situations. DTs could be utilized to 274 solve a common issue of smart cities: the siloing 275 of application domains (e.g., transportation, 276 energy, and so on). DTs offer interfaces through 277 which different applications can access data, 278 behavior, and status corresponding to specific 279 resources. DT composability enables the crea- 280 tion of object aggregations that support abstract 281 views of larger phenomena. The framework must 282 scale up from few resources to a multitude. 283 Figure 3 represents a DT platform able to cap- 284 ture the behavior of a complex system. Some 285 applications are designed to monitor the situa- 286 tion for applying policies on specific issues (traf- 287 fic, transportation, etc.). When issues arise, 288 these applications implement mechanisms to 289 minimize impacts on users and optimize 290 
resource usage. Other applications are simulations executed to understand how the "smart city" could react to changes in policies of resources' usage. Benefits of applying the DT concept to the "smart city" are many. These advantages depend upon the introduction of higher programmability levels, a strong integration with $\mathrm{AI} / \mathrm{ML}$ capabilities, the elimination of silo constraints, and the possibility to simulate the functioning of a city.

\section{BUSINESS AS USUAL?}

The DT concept has business implications. It changes the nature of POs and products. DT properties facilitate the adoption of servitization, i.e., the ability to transform a physical product into a set of services offered to the user. ${ }^{17,18}$ Servitization nurtures customization of functionalities, making products more aligned to the lifestyles and needs of people and it introduces additional innovations in the service area. ${ }^{19}$ Products could even be modified, improved, and customized by the user. New functions can be added to products if users' needs change. The customer relationship process could be transformed..$^{20}$ Customers are "entangled" with products. If malfunctions emerge in a product, they can be predicted before they actually occur and the impact on users could be mitigated. Maintenance can be improved by focusing more on preventing problems than on solving them. The products' value chain could be largely modified. As the mobile phone industry showed, a rich application layer has a tremendous impact on products and it can determine success in a global market. Customization, and the actors supporting it, could become an important component of the entire value chain of new digital markets.

\section{CONCLUSIONS}

The DT approach promises to have impact. It may provide solutions in different sectors (smart cities, e-health, e.g., virtual patient, automotive, cultural heritage), supporting the provision of servitized products, and programmability of complex systems. It fosters new ways of doing business by transforming products into sets of customizable services (an autonomous car and a for it.

smart building). A precise definition of the DT can help in better understanding requirements and impacts on IoT software platforms. DTs should be evaluated for their ability to support and deliver the expected benefits. Further investigation on how DTs can enable an ecosystem of applications that are feasible, marketable, and sustainable by the industry is needed. There are two major concerns: feasibility, i.e., the complexity and technical challenges associated with the creation of scalable, secure DT frameworks are such that the construction of a viable platform remains to be proven in-the-large; and user ownership, i.e., the possibility to frame users into a system of products and applications that limit privacy. The DT approach needs validation before it will be widely accepted and used. It already is a powerful means to control, govern, and program the lifecycle of objects, physical resources, and products, for providing better services to end-users. It is a challenge to academia and industry to find relevant applications

\section{REFERENCES}

1. M. Grieves, Product Lifecycle Management: Driving the Next Generation of Lean Thinking. New York, NY USA: McGraw-Hill, 2006.

2. R. Rosen, G. Von Wichert, G. Lo, and K. D. Bettenhausen, "About the importance of autonomy and digital twins for the future of manufacturing," IFAC-PapersOnLine, vol. 48, no. 3, pp. 567-72, Jan. 1, 2015.

3. F. Tao, J. Cheng, Q. Qi, M. Zhang, H. Zhang, and F. Sui, "Digital twin-driven product design, manufacturing and service with big data," Int. J. Adv. Manuf. Technol., vol. 94, no. 9-12, pp. 3563-3576, Feb. 1, 2018.

4. E. Negri, L. Fumagalli, and M. Macchi, "A review of the roles of digital twin in CPS-based production systems," Procedia Manuf., vol. 11, pp. 939-948, 2017.

5. S. Haag and R. Anderl, "Digital twin-proof of concept," Manuf. Lett., vol. 15, pp. 64-66, 2018.

6. C. Steinmetz, A. Rettberg, F. G. C. Ribeiro, G. Schroeder, and C. E. Pereira, "Internet of Things ontology for digital twin in cyber physical systems," in Proc. VIII Brazilian Symp. Comput. Syst. Eng., 2018, pp. 154-159. 
7. V. Souza, R. Cruz, W. Silva, S. Lins, and V. Lucena, "A digital twin architecture based on the industrial Internet of Things technologies," in Proc. IEEE Int. Conf. Consum. Electron., 2019, pp. 1-2.

8. S. Malakuti and S. Grüner, "Architectural aspects of digital twins in IloT systems," in Proc. 12th Eur. Conf. Softw. Architecture: Companion, Sep. 24, 2018, pp. 1-2.

9. S. Malakuti, J. Schmitt, M. Platenius-Mohr, S. Grüner, R. Gitzel, and P. Bihani, "A four-layer architecture pattern for constructing and managing digital twins," in Proc. Eur. Conf. Softw. Architecture, Sep. 9, 2019, pp. 231-246.

10. AIOTI - WG3 - IOT Standardization "High level architecture," Jun. 2018, Accessed on: Jan. 31, 2020. [Online]. Available: https://aioti.eu/wp-content/ uploads/2018/06/AIOTI-HLA-R4.0.7.1-Final.pdf

11. D. Happ, N. Karowski, T. Menzel, V. Handziski, and A. Wolisz, "Meeting loT platform requirements with open pub-sub solutions," Ann. Telecommun., vol. 72, no. 1/2, pp. 41-52, Feb. 2017.

12. M. Batty, "Complexity in city systems: Understanding, evolution, and design," A Planner's Encounter With Complexity. G. de Roo and E. Silva Eds. 2007, pp. 99-122.

13. S. Khan, D. Paul, P. Momtahan, and M. Aloqaily, "Artificial intelligence framework for smart city microgrids: State of the art, challenges, and opportunities," in Proc. 3rd Int. Conf. Fog Mobile Edge Comput., 2018, pp. 283-288.

14. C. Perera, A. Zaslavsky, P. Christen, and D. Georgakopoulos, "Sensing as a service model for smart cities supported by Internet of Things," Trans. Emerg. Telecommun. Technol., vol. 25, no. 1, pp. 81-93, 2014.

15. G. Garfield-Bennett, "Virtual Jersey becomes a reality," Jersey Evening Post, Feb. 8, 2019, Accessed on: Jan. 31, 2020. [Online]. Available: https:// jerseyeveningpost.com/news/business/2019/02/08/ virtual-jersey-becomes-a-reality/

16. T. Delbrügger, L. T. Lenz, D. Losch, and J. Romann, "A navigation framework for digital twins of factories based on building information modeling," in Proc. 22nd IEEE Int. Conf. Emerg. Technol. Factory Autom., 2017, pp. 1-4.

17. C. Kowalkowski, H. Gebauer, B. Kamp, and G. Parry, "Servitization and deservitization: Overview, concepts, and definitions," Ind. Marketing Manage., vol. 60, pp. 4-10, 2017.
18. Q. Qi, F. Tao, Y. Zuo, and D. Zhao, "Digital twin service 435 towards smart manufacturing," Procedia CIRP, vol. 72, 436 pp. 237-242, Jan. 1, 2018.

19. P. Zheng, T. J. Lin, C. H. Chen, and X. Xu, "A 438 systematic design approach for service innovation of 439 smart product-service systems," J. Cleaner Prod., $\quad 440$ vol. 201, pp. 657-667, Nov. 10, 2018.

20. R. N. Bolton et al., "Customer experience challenges: 442 Bringing together digital, physical and social realms," 443 J. Service Manage., vol. 29, pp. 776-808, Oct. 15, 2018.

Roberto Minerva is currently an Associate Pro- 446 fessor within the Service Architecture Lab, Institut 447 Mines Telecom-Telecom Sud Paris, Institute Poly- 448 technique de Paris, Paris, France. He received the 449 M.S. degree in computer science from Bari Univer- 450 sity, Bari, Italy, in 1987, and the Doctoral degree in 451 computer science and telecommunications from 452 UPMC (Paris-Sorbonne University) in 2013. From 453 1987 to 2016, he was a Researcher then a responsi- 454 ble of the Service Architectures and Network Intelli- 455 gence area within Telecom Italia Research Center. 456 From 2016 to 2018, he was the Technical Project 457 Leader of SoftFIRE, a European Project devoted to 458 the experimentation of NFV, SDN, and edge comput- 459 ing. He has been the Chairperson of the IEEE IOT Ini- 460 tiative in the period 2014-2016. He is a Senior 461 Member of IEEE. Contact him at roberto.minerva@te- 462 lecom-sudparis.eu.

Noël Crespi joined the Institut Mines-Telecom 464 SudParis, in 2002, and is currently a Professor and 465 Program Director with the Institut Polytechnique de 466 Paris, leading the Service Architecture Laboratory. 467 $\mathrm{He}$ is also an Adjunct Professor with KAIST 468 (South Korea), an Affiliate Professor with Concordia 469 University (Canada), and a Guest Researcher with 470 the University of Goettingen (Germany). His current 471 research interests include data analytics, the Internet 472 of Things, and softwarization. He received the mas- 473 ter's degrees from the Universities of Orsay, Paris 11, 474 France, and Kent, Canterbury, U.K., a diplome 475 d'ingénieur from Telecom ParisTech, Paris, France, 476 and the Ph.D. and Habilitation degrees from UPMC 477 (Paris-Sorbonne University). From 1993 to 2002, he 478 worked in the industrial research of telecom organi- 479 zations in the creation of new services and 480 in standardization. He is a Senior Member of IEEE. 481 Contact him at noel.crespi@mines-telecom.fr. 482 